\title{
EINE ERWEITERUNG DER CROFTONSCHEN FORMELN FÜR KONVEXE KÖRPER
}

\author{
J. BOKOWSKI, H. HADWIGER UND J. M. WILLS
}

§0. Einführung. Durch die bekannten Croftonschen Integrale können bekanntlich die Minkowskischen Quermaßintegrale konvexer Körper dargestellt werden. In der vorliegenden Note betrachten wir gewisse Erweiterungen dieser klassischen integralgeometrischen Formeln, durch die allgemeinere invariante Eikörperfunktionale gegeben sind. Es handelt sich hierbei um kinematische Integrale mit beweglichen unterdimensionalen Teilräumen, wobei passend gewählte Funktionen ihrer Abstände vom Eikörper eingehen.

$\S 0.1$. Begriffe und Bezeichnungen. Es sei $E^{n}$ der $n$-dimensionale euklidische Raum und $\mathscr{K}^{n}$ bedeute die Menge der nichtleeren, kompakten und konvexen Teilmengen $A \subset E^{n} ; A \in \mathscr{K}^{n}$ nennen wir kurz auch Eikörper. Für ein $\rho \geqslant 0$ bedeute $A_{\rho}$ den äußeren Parallelkörper von $A$ im Abstand $\rho$. Ist $E^{i} \subset E^{n}(i=0, \ldots, n)$ eine bewegliche $i$-dimensionale Ebene, so bezeichne $d E^{i}$ die Bewegungsdichte von $E^{i}$ im Sinne der Integralgeometrie (vgl. [3], S. 240). Für $A \in \mathscr{K}^{n}$ bedeute $d\left(A, E^{i}\right)$ den Abstand des Eikörpers $A$ von der Ebene $E^{i}$. Mit

$$
\omega_{i}=\frac{\pi^{i / 2}}{\Gamma(1+i / 2)} \quad(i=0, \ldots, n)
$$

ist das Volumen der $i$-dimensionalen Einheitskugel gegeben. Wir benötigen noch die integralgeometrischen Hilfskonstanten

$$
c_{0 n}=1 \quad \text { und } \quad c_{i n}=\left(\begin{array}{c}
n \\
i
\end{array}\right) \frac{\omega_{n-1} \ldots \omega_{n-i}}{\omega_{1} \ldots \omega_{i}} \quad(i=1, \ldots, n) .
$$

§0.2. Definition erweiterter Croftonscher Integrale. Sei $f(r)$ eine in $[0, \infty)$ definierte nichtnegative, lokal integrierbare Funktion, für die $f(0) \neq 0$ vorausgesetzt und die Existenz der ersten $n$ Integralmomente

$$
M_{k}(f)=\int_{0}^{\infty} f(r) r^{k} d r \quad(k=0, \ldots, n-1)
$$

gefordert wird. Mit $\mathscr{F}$ werde die Klasse aller Funktionen $f$ mit diesen Eigenschaften bezeichnet. Ist $A \in \mathscr{K}^{n}$ ein Eikörper und $r=d\left(A, E^{i}\right), f \in \mathscr{F}$, so werden mit den Ansätzen

$$
P_{n}(f, A)=f(0) \omega_{n} ; P_{i}(f, A)=\frac{1}{c_{i n}} \int f(r) d E^{i} \quad(i=0, \ldots, n-1),
$$

wobei sich die Integration über alle Lagen der Ebene $E^{i}$ im Raum $E^{n}$ zu erstrecken hat, $n+1$ Eikörperfunktionale $P_{i}(f, A)(i=0, \ldots, n)$ definiert. Mit der speziellen 
Funktion $f_{w}(r)=1(r=0), 0(r>0)$ resultieren die Croftonschen Integrale und es gilt

$$
P_{i}\left(f_{w}, A\right)=W_{i}(A) \quad(i=0, \ldots, n),
$$

so daß unsere Funktionale $P_{i}$ also die Minkowskischen Quermaßintegrale $W_{i}$ darstellen. In diesem Sinne stellen die Integrale (0.2.2), deren Existenz für alle $f \in \mathscr{F}$ nachgewiesen werden kann, erweiterte Croftonsche Formeln dar.

\section{§1. Ergebnisse.}

§1.1. Allgemeine Ergebnisse. Ein erstes relevantes Ergebnis ist mit

$$
P_{i}(f, A)=\sum_{k=0}^{n-i}\left(\begin{array}{c}
n-i \\
k
\end{array}\right) C_{k}(f) W_{i+k}(A) \quad(i=0, \ldots, n)
$$

gegeben, mit

$$
C_{0}(f)=f(0), C_{k}(f)=k M_{k-1}(f) \quad(k=1, \ldots, n) .
$$

Wie gezeigt werden wird, bilden die $P_{i}(f, A)(i=0, \ldots, n)$ für jedes feste $f \in \mathscr{F}$ eine Basis im Raum $\mathscr{R}^{n}$ der auf $\mathscr{K}^{n}$ erklärten reellwertigen bewegungsinvarianten, additiven und stetigen Funktionale. Damit Jassen sich speziell die Quermaßintegrale $W_{i}$ bei gegebenem $f$ als Linearkombination der $P_{i}$ ausdrücken. In der Tat gilt die Darstellungsformel

$$
W_{i}(A)=\sum_{k=0}^{n-i}\left(\begin{array}{c}
n-i \\
k
\end{array}\right) \tilde{C}_{k}(f) P_{i+k}(f, A) \quad(i=0, \ldots, n),
$$

wobei die benötigten Koeffizienten durch

$$
\frac{1}{g(x)}=\sum_{k=0}^{\infty} \tilde{C}_{k}(f) \frac{x^{k}}{k !}
$$

erzeugt werden und die Hilfsfunktion mit

$$
g(x)=\sum_{k=0}^{n} C_{k}(f) \frac{x^{k}}{k !}
$$

gegeben ist.

Ein weiteres Resultat ist mit einer Verallgemeinerung des vollständigen Steinerschen Formelsystems (vgl. [3], S. 214) gegeben, wonach

$$
P_{i}\left(f, A_{\rho}\right)=\sum_{k=0}^{n-i}\left(\begin{array}{c}
n-i \\
k
\end{array}\right) P_{i+k}(f, A) \rho^{k} \quad(i=0, \ldots, n)
$$

gilt. Ferner läßt sich die Integraldarstellung

$P_{i}(f, A)=f(0) W_{i}(A)+(n-i) \int_{0}^{\infty} W_{i+1}\left(A_{\rho}\right) f(\rho) d \rho \quad(i=0, \ldots, n)$

verifizieren.

Aufgrund der Darstellung (1.1.1) lassen sich viele Eigenschaften der Quermaßintegrale $W_{i}$ unmittelbar auf die Funktionale $P_{i}$ übertragen; lediglich die Homogenität 
geht i.a. verloren. Neben der selbstverständlichen Bewegungsinvarianz sind aber Additivität, Monotonie und Stetigkeit besonders hervorzuheben. Auch das Verhalten bei Symmetrisierungen ist das nämliche.

\$1.2. Ergebnisse bei spezieller Wahl von $f \in \mathscr{F}$. Mit $a>0$ setzen wir zunächst $f(r)=f_{a}(r)=e^{-r / a}$. Es ist $M_{k-1}\left(f_{a}\right)=(k-1) ! a^{k}(k \geqslant 1)$ und nach (1.1.1) resultiert mit $f(0)=1$

$$
P_{i}\left(f_{a}, A\right)=\sum_{k=0}^{n-i}\left(\begin{array}{c}
n-i \\
k
\end{array}\right) k ! a^{k} W_{i+k}(A) \quad(i=0, \ldots, n) .
$$

Beachtenswert ist, daß die Inversionsformel (1.1.2) im vorliegenden Fall eine ganz besonders einfache Gestalt annimmt. Es gilt

$$
W_{i}(A)=P_{i}\left(f_{a}, A\right)-(n-i) a P_{i+1}\left(f_{a}, A\right) \quad(i=0, \ldots, n) .
$$

Ein weiterer Sonderfall ist mit $a>0$ mit dem Ansatz

$$
f(r)=g_{a}(r)=a(r=0), 1 / a(0<r<a), 0(a \leqslant r<\infty)
$$

vorliegend. Die Absicht besteht darin, mit dem Grenzuibergang $a \rightarrow 0$, wobei der nicht existierenden Grenzfunktion $g$ die Wirkung einer " Diracfunktion " zugebilligt werden soll, ein invariantes $\mathrm{Maß}$ für die Menge derjenigen $E^{i} \mathrm{zu}$ gewinnen, die $A$ berühren. Diese Deutung wird plausibel, wenn das zuständige Integral $(0.2 .2)$ in der Form

$$
P_{i}\left(g_{a}, A\right)=a W_{i}(A)+\frac{1}{a c_{i n}} \int_{0<r<a} d E^{i} \quad(i=0, \ldots, n-1)
$$

geschrieben wird. Mit der Bemerkung $M_{k-1}\left(g_{a}\right) \rightarrow 1(k=1), 0(k>1)$ mit $a \rightarrow 0$ folgt nach (1.1.1)

$$
P_{i}\left(g_{a}, A\right) \rightarrow(n-i) W_{i+1}(A) \quad(a \rightarrow 0 ; i=0, \ldots, n-1) .
$$

Abgesehen von der unwesentlichen Normierung stellt $W_{i+1}(A)$ in der Tat das in Aussicht gestellte Berührmaß des Eikörpers $A$ durch $i$-dimensionale Ebenen dar, wie dies von J. Zelver [6] angegeben worden ist. Eine allgemeinere Theorie invarianter Berührmaße wurde von W. J. Firey [2] begründet.

\$1.3. Ergebnisse im Eall $i=0$. Mit der Restriktion $i=0$ beschränken wir uns im weiteren lediglich auf Punkte $E^{0}$, werden aber anstelle einer Funktion $f$ ein System von $n+1$ Funktionen $f_{\mu} \in \mathscr{F}(\mu=0, \ldots, n)$ miteinbeziehen. Aus der Vielfalt der sich anbietenden Möglichkeiten wählen wir den Fall

$$
f_{\mu}(r)=r^{2 \mu} e^{-\pi r^{2}} \quad(\mu=0, \ldots, n)
$$

aus, der in einem weiter unten angedeuteten Zusammenhang besondere Beachtung verdient. Es ergeben sich die Funktionale

$$
H_{\mu}(A)=P_{0}\left(f_{\mu}, A\right)=\int r^{2 \mu} e^{-\pi r^{2}} d p \quad(\mu=0, \ldots, n),
$$

wo $d E^{0}=d p$ (Punktdichte) gesetzt wurde. Anschließend an (1.1.1) erhält man mit 
direkter Rechnung

$$
H_{\mu}(A)=\sum_{\nu=0}^{n} C_{\mu v}\left(\begin{array}{l}
n \\
v
\end{array}\right) \frac{1}{\omega_{v}} W_{v}(A) \quad(\mu=0, \ldots, n),
$$

wobei

$$
C_{0 v}=1 ; C_{\mu v}=(2 \pi)^{-\mu} v(v+2) \ldots(v+2 \mu-2) \quad(v=0, \ldots, n ; \mu=1, \ldots, n)
$$

ist. Im speziellen Fall $\mu=0$ ergibt sich das Funktional $H_{0}(A)=W(A)(\operatorname{vgl}$. [4]), nämlich

$$
W(A)=\sum_{v=0}^{n}\left(\begin{array}{l}
n \\
v
\end{array}\right) \frac{1}{\omega_{v}} W_{v}(A),-
$$

das in der Geometrie der Zahlen im Zusammenhang mit Fragen der Gitterpunktszahl in Eikörpern Gegenstand intensiver Studien geworden ist.-Es sollen noch einige Eigenschaften der Funktionale $H_{\mu}$ angegeben werden. Seien $A, B \in \mathscr{K}^{n}, A \subset E^{i}$, $B \subset E^{n-i}$, wobei $E^{i}$ und $E^{n-i}$ zwei komplementäre totalorthogonale Teilräume von $E^{n}$ anzeigen. Für den durch Minkowskische Addition aus $A$ und $B$ hervorgehenden Körper $A+B$ gilt dann

$$
H_{\mu}(A+B)=\sum_{v=0}^{\mu}\left(\begin{array}{l}
\mu \\
v
\end{array}\right) H_{v}{ }^{\prime}(A) H_{\mu-v}^{\prime \prime}(B) \quad(\mu=0, \ldots, n),
$$

wo $H_{v}{ }^{\prime}$ und $H^{\prime \prime}{ }_{\mu-\vartheta}$ die sich auf die Trägerräume $E^{i}$ und $E^{n-i}$ beziehenden Funktionale anzeigen sollen.-Sei ferner $A \in \mathscr{K}^{n}, A \subset E^{n-1}$ und bezeichnet $H_{v}{ }^{\prime}$ wieder das auf den $E^{n-1}$ bezogene Funktional, so gilt

$$
H_{\mu}(A)=\sum_{v=0}^{\mu} C_{(\mu-v) 1}\left(\begin{array}{l}
\mu \\
v
\end{array}\right) H_{v}^{\prime}(A) \quad(\mu=0, \ldots, n)
$$

wenn die in (1.3.3) definierten Koeffizienten $C_{\mu \nu}$ benutzt werden. Mit $C_{\mu \nu} \geqslant 0$ läßt sich der Darstellung (1.3.3) entnehmen, daß die Funktionale $H_{\mu}$ bezüglich SteinerSymmetrisierung, also auch bei der Schwarzschen Abrundung und Kugelung nicht vergrößert werden. Analoges gilt für die Blaschkesche Symmetrisierung und bei Zentralsymmetrisierung.-Geht $A$ durch die Steinersche Symmetrisierung in $A^{\prime}$ über, so kann die Aussage

$$
\begin{aligned}
0 \leqslant \frac{2 \mu+1}{2 \pi}\left(H_{\mu}(A)-\right. & \left.H_{\mu}\left(A^{\prime}\right)\right) \\
& \leqslant H_{\mu+1}(A)-H_{\mu+1}\left(A^{\prime}\right) \quad(\mu=0, \ldots, n-1)
\end{aligned}
$$

gemacht werden.-Schließlich sei vermerkt, daß die $H_{\mu}(\mu=0, \ldots, n)$ wieder eine Basis im Raum $R\left(\mathscr{K}^{n}\right)$ bilden, so daß sich analog zu (1.1.2) die Quermaßintegrale $W_{v}$ als Linearformen der $H_{\mu}$ darstellen lassen. Für die Inversion des Systems (1.3.3) zuständige Determinante ergibt sich der positive Wert

$$
\operatorname{det} C_{\mu \nu}=(2 \pi)^{-\left(\begin{array}{c}
n+1 \\
2
\end{array}\right)} 1 ! 2 ! \ldots n !
$$


§2. Beweise.

$\S 2.1$. 1. Die Feststellung (0.2.3) resultiert mit der bekannten integralgeometrischen Formel (vgl. [3], S. 240 (120))

$$
W_{i}(A)=\frac{1}{c_{\text {in }}} \int d E^{i}
$$

wobei sich die Integration über alle $E^{i}$ mit $A \cap E^{i} \neq \varnothing$ erstreckt.--Das Ergebnis (1.1.1) ist nach Ansatz (0.2.2) für $i=n$ trivial. Wir führen den Nachweis im übrigen zunächst für $i=0$. Nach $(0.2 .2)$ ist $P_{0}(f, A)=\int f(r) d p$, wenn statt $d E^{0}$ die Punktdichte $d p$ geschrieben wird. Mit naheliegender Umdeutung läßt sich

$$
P_{0}(f, A)=f(0) V(A)+\int_{0}^{\infty} f(r) F\left(A_{r}\right) d r
$$

setzen, wenn $A_{r}$ den Parallelkörper von $A$ und $F$ die Oberfläche anzeigen. Nach der Steinerschen Formel (vgl. [3], S. 214 (49)) ist

$$
F\left(A_{r}\right)=\sum_{k=1}^{n}\left(\begin{array}{l}
n \\
k
\end{array}\right) k W_{k}(A) r^{k-1},
$$

so daß also

$$
P_{0}(f, A)=f(0) V(A)+\sum_{k=1}^{n}\left(\begin{array}{l}
n \\
k
\end{array}\right) k M_{k-1}(f) W_{k}(A)
$$

resultiert, wzzw.-Sei nun $1 \leqslant i \leqslant n-1$. Wir spalten die kinematische Dichte $d E^{i}=d \bar{E}^{i} d \bar{E}^{i}$ in die Translationsdichte $d \bar{E}^{i}$ und die Drehdichte $d \bar{E}^{i}$ auf (vgl. [3], S. 227). Unter allen Drehlagen halten wir zunächst eine fest und führen die Integration nur über die Translationen aus. Die Translationsdichte $d \bar{E}^{i}$ läßt sich deuten als Punktdichte $d p^{\prime}$ in dem zu allen jetzt zu betrachtenden parallelen Ebenen $E^{i}$ total orthogonalen $(n-i)$-dimensionalen Raum $E^{n-i}$. Da die Drehdichten $d \bar{E}^{i}$ und $d \bar{E}^{n-i}$ übereinstimmen, erhält man so

$$
P_{i}(f, A)=\frac{1}{c_{i n}} \iint f(r) d p^{\prime} d \bar{E}^{n-i},
$$

wobei sich die innere Integration über $E^{n-i}$ erstreckt. Da $r=d\left(A, E^{i}\right)=d\left(A^{\prime}, E^{\dagger}\right)$ ist, wo $A^{\prime}$ den Normalriss von $A$ in $E^{n-i}$ bezeichnet, ergibt sich

$$
P_{i}(f, A)=\frac{1}{c_{\text {in }}} \int P_{0}^{\prime}\left(f, A^{\prime}\right) d \bar{E}^{n-i},
$$

wobei $P_{0}^{\prime}$ das entsprechende Funktional im $E^{n-i}$ bedeutet. Mit dem oben für $i=0$ sichergestellten Ergebnis ist

$$
P_{0}^{\prime}\left(f, A^{\prime}\right)=f(0) V^{\prime}\left(A^{\prime}\right)+\sum_{k=1}^{n-i}\left(\begin{array}{c}
n-i \\
k
\end{array}\right) k M_{k-1}(f) W_{k}^{\prime}\left(A^{\prime}\right)
$$


so daß sich mit Verwendung der Cauchyschen Projektionsformel (vgl. [3], S. 232 (102)), wonach

$$
\frac{1}{c_{\text {in }}} \int W_{k}^{\prime}\left(A^{\prime}\right) d \bar{E}^{n-1}=W_{i+k}(A)
$$

für $k=0, \ldots, n-i$ gilt, schließlich mit $W_{0}{ }^{\prime}\left(A^{\prime}\right)=V^{\prime}\left(A^{\prime}\right)$

$$
P_{i}(f, A)=f(0) W_{i}(A)+\sum_{k=1}^{n-i}\left(\begin{array}{c}
n-i \\
k
\end{array}\right) k M_{k-1}(f) W_{i+k}(A)
$$

ergibt, womit (1.1.1) vollständig bewiesen ist. Es sei noch beigefügt, daß die durchgeführten Rechnungen implizite auch den Existenzbeweis für die mit (0.2.2) angesetzten Integrale enthalten.

Da die Matrix des linearen Systems (1.1.1) Dreiecksgestalt aufweist und die Hauptdiagonalglieder nicht verschwinden, ist diese invertierbar. Eine explizite Darstellung der inversen Matrix und also der Auflösung des Systems (1.1.1) nach den $W_{v}$ hat G. Wieczorek [5] durchgeführt.

Mit Hilfe einer Formel von Faà di Bruno (vgl. [1], S. 823) ergibt sich unsere Auflösungsformel (1.1.2), die in diesem Sonderfall leicht direkt verifizierbar ist. Ersetzt man dort die $P_{i+k}$ gemäß (1.1.1) und nutzt die aus der Erzeugung der $\widetilde{C}_{k}$ resultierende Beziehung

$$
\sum_{v=0}^{\mu}-\frac{1}{v !(\mu-v) !} \tilde{C}_{v}(f) C_{\mu-v}(f)=\left\{\begin{array}{l}
1(\mu=0), \\
0(\mu=1, \ldots, n),
\end{array}\right.
$$

so ergibt sich $W_{i}$, womit (1.1.2) bestätigt ist.-

Verwendet man die Steinersche Formel (vgl. [3], S. 214)

$$
W_{m}\left(A_{\rho}\right)=\sum_{v=0}^{n-m}\left(\begin{array}{c}
n-m \\
v
\end{array}\right) W_{v+m}(A) \rho^{v}
$$

in (1.1.1), so ergibt sich zunächst

$$
P_{i}\left(f, A_{\rho}\right)=\sum_{k=0}^{n-i} \sum_{\mu=0}^{n-i-k}\left(\begin{array}{c}
n-i \\
k
\end{array}\right)\left(\begin{array}{c}
n-i-k \\
\mu
\end{array}\right) C_{k}(f) W_{\mu+i+k}(A) \rho^{\mu}
$$

und mit Vertausch der Summationsreihenfolge und Berücksichtigung von

$$
\left(\begin{array}{c}
n-i \\
k
\end{array}\right)\left(\begin{array}{c}
n-i-k \\
\mu
\end{array}\right)=\left(\begin{array}{c}
n-i-\mu \\
k
\end{array}\right)\left(\begin{array}{c}
n-i \\
\mu
\end{array}\right)
$$

schließlich

$$
P_{i}\left(f, A_{\rho}\right)=\sum_{\mu=0}^{n-i}\left(\begin{array}{c}
n-i \\
\mu
\end{array}\right) P_{i+\mu}(f, A) \rho^{\mu} .
$$

Damit ist (1.1.3) bewiesen. Die Darstellung (1.1.4) ergibt sich aus (1.1.1) unter Verwendung des Steinerschen Formelsystems. 
§2.1.2. Die Bestätigung von (1.2.2) mit Verwendung von (1.2.1) gelingt mühelos. Alle Aussagen dieses Teils sind durch die im Text enthaltenen Hinweise ausreichend klar gemacht.

$\S 2.1 .3$. Wird in (1.1.1) $i=0$ gesetzt, so ergibt sich

$$
P_{0}(f, A)=f(0) V(A)+\sum_{v=1}^{n}\left(\begin{array}{l}
n \\
v
\end{array}\right) v M_{v-1}(f) W_{v}(A) .
$$

Für $f=f_{\mu}$ ist $f(0)=1(\mu=0), 0(1 \leqslant \mu \leqslant n)$ zu beachten und für $1 \leqslant v \leqslant n$ das Resultat

$$
v M_{\nu-1}\left(f_{\mu}\right)=v \int_{0}^{\infty} r^{\nu+2 \mu+1} e^{-\pi r^{2}} d r=\frac{v}{(v+2 \mu) \omega_{v+2 \mu}}
$$

zu verwenden. Damit folgt

$$
\begin{gathered}
H_{\mu}(A)=\sum_{v=1}^{n}\left(\begin{array}{l}
n \\
v
\end{array}\right) \frac{v}{(v+2 \mu) \omega_{v+2 \mu}} W_{v}(A) \quad(1 \leqslant \mu \leqslant n) \\
H_{0}(A)=V(A)+\sum_{v=1}^{n}\left(\begin{array}{l}
n \\
v
\end{array}\right) \frac{1}{\omega_{v}} W_{v}(A) \quad(\mu=0)
\end{gathered}
$$

Benutzen wir noch die Rekursion

$$
(2 \mu+v)(2 \mu+v-2) \ldots(v+2) \omega_{v+2 \mu}=(2 \pi)^{\mu} \omega_{v} \quad(\mu \geqslant 1),
$$

so gelangen wir zur Formel (1.3.3), welche die beiden oben angegebenen Fälle bezüglich $\mu$ zusammenfaßt. Hierbei ist noch $V=W_{0}$ zu bedenken. Wir wenden uns nun der Aussage (1.3.5) zu. Sind $p^{\prime}=p\left|E^{i}, p^{\prime \prime}=p\right| E^{n-i}$ die Lotpunkte von $p$ auf $E^{i}$ bzw. $E^{n-i}$, so gilt für die Abstände

$$
r(p, A+B)^{2}=r\left(p^{\prime}, A\right)^{2}+r\left(p^{\prime \prime}, B\right)^{2}
$$

und für die Punktdichten $d p=d p^{\prime} d p^{\prime \prime}$. Verwenden wir dies im Integral

$$
H_{\mu}(A+B)=\int r^{2 \mu} e^{-\pi r^{2}} d p,
$$

so resultiert mit binomischer Entwicklung der Potenz

$$
H_{\mu}(A+B)=\sum_{\nu=0}^{\mu}\left(\begin{array}{l}
\mu \\
v
\end{array}\right) H_{v}{ }^{\prime}(A) H^{\prime \prime}{ }_{\mu-v}(B)
$$

wzzw. Hier ist zu beachten, daß $v>i$ sein kann, ebenso $\mu-v>n-i$; die $H_{v}{ }^{\prime}$ bzw $H^{\prime \prime}{ }_{\mu-\nu}$ sind dann linear abhängig.-Weiter ist (1.3.6) ein Korollar zu (1.3.5). Wird dort $B=0$ (Ursprungspunkt) gesetzt, so kann $0 \subset E^{1}$ als uneigentlicher Eikörper in dem zu $E^{n-1}$ orthogonal stehenden Raum $E^{1}$ gedeutet werden. Nach (1.3.3) folgt mit $n=1, W_{0}{ }^{\prime \prime}(0)=0, W_{1}{ }^{\prime \prime}(0)=\omega_{1}$ der Tatbestand $H^{\prime \prime}{ }_{\mu-v}(0)=C_{(\mu-v) 1}$, so daß sich mit (1.3.5) also (1.3.6) ergibt, wenn man noch $A+0=A$ bedenkt.-

Um (1.3.7) nachzuweisen, greifen wir auf die Darstellung (1.3.3) und erhalten 
mit Rücksicht auf $W_{0}(A)=W_{0}\left(A^{\prime}\right)$ zunächst

$$
H_{\mu+1}(A)-H_{\mu+1}\left(A^{\prime}\right)=\sum_{v=1}^{n} C_{(\mu+1) v}\left(\begin{array}{l}
n \\
v
\end{array}\right) \frac{1}{\omega_{v}}\left(W_{v}(A)-W_{v}\left(A^{\prime}\right)\right)
$$

und sodann mit $C_{(\mu+1) v} \geqslant((2 \mu+1) / 2 \pi) C_{\mu v}$ unmittelbar die zu beweisende Ungleichung.

Endlich begründen wir die Aussage (1.3.8). Wegen $C_{\mu 0}=0$ für $\mu=1, \ldots, n$ und $C_{00}=1$ ist $\operatorname{det} C_{\mu v}=(2 \pi)^{-n(n+1) / 2} \operatorname{det} A_{\mu v}$ mit $A_{\mu v}=v(v+2) \ldots(v+2 \mu-2)$ $(\mu, v=1, \ldots, n)$. Wegen $A_{\mu \nu}-2(\mu-1) A_{(\mu-1) v}=v A_{(\mu-1) v}(\mu=2, \ldots, n)$ kann für $\mu=2, \ldots, n$ in der $\mu$-ten Zeile $A_{\mu \nu}$ durch $v A_{(\mu-1) v}$ ersetzt werden; d.h. anstelle des Faktors $v-2 \mu-2$ tritt $v$. Analog ist $A_{(\mu-1) v}-2(\mu-2) A_{(\mu-2) v}=v A_{(\mu-2) v}$ $(\mu=3, \ldots, n)$, und so kann für $\mu=3, \ldots, n$ in der $\mu$-ten Zeile der Faktor $v+2 \mu-4$ durch $v$ ersetzt werden, usw. So erhält man

$$
\operatorname{det} A_{\mu v}=\operatorname{det} v^{\mu}=D_{n} \quad(\mu, v=1, \ldots, n) .
$$

Wenn aus der $v$-ten Spalte der Faktor $v$ vorgezogen wird und dies für alle $v=1, \ldots, n$, so ergibt sich

$$
D_{n}=n ! \operatorname{det} v^{\mu-1}(\mu, v=1, \ldots, n) .
$$

Nun ist det $v^{\mu-1}$ eine spezielle Vandermondesche Determinante mit dem bekannten Wert $1 ! \ldots(n-1)$ !, so daß sich $D_{n}=1 ! \ldots n !=n !$ ! ergibt. Damit ist $(1.3 .8)$ nachgewiesen.

\section{Literatur}

1. Abramowitz and Stegun. Handbook of Mathematical Functions.

2. W. J. Firey. "An integral-geometric meaning for lower order area functions of convex bodies", Mathematika, 19 (1972), 205-212.

3. H. Hadwiger. Vorlesungen über Inhalt, Oberfäche und Isoperimetrie (Springer; Berlin-GöttingenHeidelberg, 1957).

4. H. Hadwiger. "Das Wills'sche Funktional", Mh. Math., 79 (1975), 213-221.

5. G. Wieczorek. " Integralgeometrische Darstellungen von Linearkombinationen Minkowskischer Quermassintegrale", Diplomarbeit Technische Universität (Berlin, 1975).

6. J. Zelver. "The integro-geometric tangent measures of Euclidean $n$-space", Ph.D. Thesis (Oregon State University, Corvallis, 1970).

Mathematisches Institut,

Ruhr-Universität,

Buscheystraße,

4630 Bochum,

Deutschland.

Mathematisches Institut, Universität Bern,

Sidlerstraße 5,

3000 Bern,

Schweiz.

Lehrstuhl für Mathematik II,

Gesamthochschule Siegen,

Hölderlinstr. 3,

5930 Hüttental-Weidenau,

Deutschland.
28A75: MEASURE AND INTEGRATION; Geometric measure theory.

52A20: CONVEX SETS AND GEOMETRIC INEQUALITIES; Convex sets in $n$ dimensions.

Received on the 29th of September, 1975. 